\title{
Human Papillomavirus Infection in Rio de Janeiro, Brazil: a Retrospective Study
}

\author{
Carvalho M.O.O., Carestiato F.N., Perdigão P.H., \\ Xavier M.P.P.T., Silva KC., Botelho M.O., \\ Oliveira L.H.S. and Cavalcanti S.M.B.
}

\author{
Departmemt of Microbiology and Parasitology, Biomedical \\ Institute of Universidade Federal Fluminense, Niterói/RJ and \\ Department of Molecular Biology, Laboratórios Sérgio Franco; \\ Rio de Janeiro, RJ, Brazil
}

\begin{abstract}
There is considerable data to support a central role for human papillomavirus (HPV) in the etiology of cervical cancer. More than a 100 HPV types have been described, and 40 have been isolated from benign and malignant genital lesions. Consequently, there is strong motivation to evaluate HPV testing for cervical cancer screening. Few studies concerning the natural history of HPV infection have been conducted in the state of Rio de Janeiro. We determined the prevalence of HPV types in female genital lesions by using Hybrid Capture Assay (HCA) and we retrospectively analyzed the course of HPV infection. Our sample included 788 women attended at Laboratórios Sérgio Franco. The average age of the participants was 29.6 years. HPV prevalence and cytological diagnosis were determined. The overall prevalence of HPV DNA in the study group was $\mathbf{5 0 . 1 \%}$ (395/788), ranging from $25 \%$ (NORMAL) to $100 \%$ in high-grade intraepithelial lesions (HSIL). High risk HPV was found in $12 \%$ inflammatory, 58.3\% HPV, 63.2\% LSIL and 100\% HSIL. A retrospective analysis of 78 patients showed that 22 presented persistent lesions, 2 had progressive lesions, 4 had regressive lesions, 13 showed latent infections, 18 were transiently infected and 19 were submitted to curative treatment. No cases of cancer were registered in this population, which can afford private medical care and regular follow-up exams. We suggest that HCA be used in specific cases involving persistent and recurrent lesions.

Key Words: HPV, cervical cancer, Rio de Janeiro, Brazil.
\end{abstract}

The extensive experimental and epidemiological data that has been accumulated support the hypothesis that human papillomavirus (HPV) plays a central role in the etiology of anogenital cancer, especially cervical carcinoma[1]. Nearly 100 different types of HPV have been described, and 40 of these have been isolated from benign and malignant genital lesions [2]. These viruses have been classified according to their malignant potential. High risk types (e. g. HPV 16, 18, 31, 33, and 35) have been linked to high-grade intraepithelial lesions (HSIL) and invasive carcinoma, whereas low-

Received on 17 June 2005; revised 12 September 2005.

Address for correspondence: Dr. Silvia Cavalcanti. Departamento de Microbiologia e Parasitologia, Instituto Biomédico, Universidade Federal Fluminense, Rua Ernani Melo, 101, Zip code: 24210-030, Rio de Janeiro, RJ, Brazil. Email: silviabc@ism.com.br.

The Brazilian Journal of Infectious Diseases 2005;9(5):398-404 (C) 2005 by The Brazilian Journal of Infectious Diseases and Contexto Publishing. All rights reserved. risk types (e.g. HPV 6 and 11) have been associated with benign or low-grade SIL (LSIL) [3]. Differences in oncogenic potential of low and high-risk types have also been demonstrated by transformation experiments [4].

Early detection of oncogenic HPV types in precursor lesions would help determine adequate treatment and subsequent follow-up. Consequently, there is strong motivation to evaluate HPV testing for cervical cancer screening, which requires further improvements in and standardization of testing methods. HPV detection has generally been conducted by Southern blot and PCR methods. But neither research assays nor commercial kits (dot blot or in situ hybridization) have been found to be adequate for clinical use. An assay for routine clinical use requires reliable and accurate detection of the broad range of pathogenic HPV types that infect the genital tract [5].

Recently developed, the second generation of the Hybrid Capture System HPV DNA detection test from 
Digene Diagnostics (Silver Spring, Md.) is a nonradioactive, relatively rapid, liquid hybridization assay designed to detect 18 HPV types, divided into highand low-risk groups. This assay kit detects high-risk types 16, 18, 31, 33, 35, 39, 45, 51, 52, 56, 58, 59 and 61 . The low-risk group that it detects involves the types more commonly associated with condyloma acuminatum: HPV types 6, 11, 42, 43 and 44 [6].

Cervical cancer is the second-leading cause of deaths in women worldwide. In many developing countries, it is still the main cause of death from cancer in women [5]. High annual cancer incidences have been reported in Brazil. Nevertheless, there have been few published epidemiological studies concerning the prevalence of HPV DNA in the female genital tract and the natural history of the infection [7]. Vaccine trials against HPV are underway, and one of the problems in vaccine development is to accurately determine which types of HPV to include, since geographical variations in the prevalence of high-risk types have been observed [8].

We examined the prevalence of different HPV types in squamous intraepithelial lesions of the cervix of Brazilian women, correlating it with cytological data and, retrospectively, with the clinical course of infection, in order to provide tools to interpret the natural history of HPV infection and the related risk of cancer progression in the female population. These data may assist the public health authorities in planning prophylactic and therapeutic strategies to prevent cervical cancer.

\section{Material and Methods}

\section{$\underline{\text { Study population and collection of specimens }}$}

The study population included 788 women attended at Laboratórios Sérgio Franco, Rio de Janeiro, from 1998 to 2000. Laboratórios Sérgio Franco is a private service that works cooperatively with the Fluminense Federal University in Rio de Janeiro. Women were referred for routine exam. The cervical smears were collected by using a cervical cytobrush; they were transported in Digene Specimen Transport Medium
(Digene Diag, Md). A structured questionnaire was applied after informed consent was obtained. Tests were conducted at the Sérgio Franco laboratory by an expert and accompanied by a master's student from the Pathology Service at the Universidade Federal Fluminense(UFF). This study (part of a master's thesis) was approved by the Ethics Comitee of the UFF.

\section{Cytological testing}

The Papanicolaou test was applied, and smears were classified as NORMAL for normal epithelium, inflammatory for minor alterations of cervical cells, HPV for viral presence with no lesion, LSIL for lowgrade squamous intraepithelial lesions, HSIL for highgrade squamous intraepithelial lesions, in situ carcinoma, and invasive cancer.

\section{HPV testing}

Following the kit protocol, specimens were treated with sodium hydroxide to hydrolyze specimen RNA and denature the DNA. The liberated single-strand DNA was hybridized in a solution containing an RNAprobe mix, consisting of the high-risk or the low-risk HPV types. Each reaction mixture, containing any RNA-DNA hybrids that formed, was transferred to a capture tube coated with antibodies specific to the hybrids, immobilizing them. Bound RNA-DNA hybrids were then bound with an alkaline phosphataseconjugated antibody directed against the hybrids. Nonreacting material was removed by washing, and a dioxetane-based chemiluminescent compound, LumiPhos 530, was added as a substrate for alkaline phosphatase. The light produced by the ensuing reaction was measured with a Luminometer. Light measurements were expressed as relative light units (RLU). Sonicated herring sperm DNA in Digene transporting medium $(100 \mathrm{~g} / \mathrm{mL})$ was used as a negative control. Triplicate specimens of HPV 16 or HPV 11 DNA at 10pg/ served as the positive controls for highrisk and low-risk probes, respectively.

All RLU measurements for specimens were divided by the mean RLU of the three appropriate positive 
controls (PCs) to give a ratio of specimen RLU/PC. A ratio of 1.0 or greater was regarded as positive for HPV DNA, and a ratio of less than 1.0 was regarded as negative.

\section{$\underline{\text { Statistical analysis }}$}

The statistical significance of the results was analyzed by using the $x^{2}$ test for heterogeneity with the Yates continuity correction. Analyses were made using Epi Info 6.02 (CDC).

\section{Results}

Seven hundred and eighty-eight female cervical samples were investigated to detect the presence of HPV DNA. The average age of participants was 29.6 years. Three hundred and eighty one out of the 788 (48.4\%) women were from 21 to 30 years old. A similar profile was observed for infected patients (199/395) (50.4\%) confirming this age interval as the most affected by HPV infection ( $<<0.0001$, Tables 1 and 2$)$.

The HPV DNA prevalence was compared with the cytological diagnosis (PAP test, Table III); the overall prevalence of HPV DNA in the study group was 50.1\% (395/788), ranging from 25\% (NORMAL) to 100\% (HSIL). Statistically significant differences were detected between thepercentages of individuals with HPV infection of the cervix in NORMAL versus HSIL $(p<0.0001)$.

The prevalence of the different HPV types in the different kinds of lesions was compared (Table 3). Lowrisk types (Group A) were detected in $11.0 \%$ of the inflammatory cytology samples, while in HSIL, no lowrisk HPV was found alone. High-risk HPV (Group B) was found in $12 \%$ of the inflammatory, $58.3 \%$ of the HPV, $63.2 \%$ of the LSIL and $100 \%$ of the HSIL. Multiple infections (group A + B) were detected in $33.2 \%(131 / 395)$ of the positive samples.

In a retrospective analysis (24 months) of 78 patients, 22 women (28\%) presented persistent lesions caused by high-risk HPV in 82\% of the cases; among them, 2 showed progressive lesions with high-risk HPV alone or in mixed infections, 4 had regressive lesions with 50\% oncogenic HPV, 13 presented latent infections (normal smears), caused by high-risk HPV in $77 \%$ of the cases, 18 (23\%) were transiently infected by low risk (44\%) and high risk types (39\%) and mixed infections with both viruses in $17 \%$ of the cases. Finally, 19 were submitted to curative treatment, $78 \%$ caused by low risk HPV (Table 4).

\section{Discussion}

Hybrid Capture assay has proved to be a reliable, accurate, and cost-effective method for HPV testing in routine clinical practice [5]. Hence, we used this methodology to check for the presence of HPV in 788 cervical smears from female patients resident in the state of Rio de Janeiro. Our population was attended at a private laboratory, which cooperates with diverse medical care institutes. These individuals belong to an intermediate economic class that can afford private medical care services.

The overall prevalence of HPV DNA in the study group was $50.1 \%$ (395/788). Among the 395 positive women, 32 (8.1\%) were infected with low-risk HPV types (Group A), 232 (58.7\%) had high-risk HPV types (Group B) and 131 (33.2\%) had both groups. Hence, 363 (91.8\%) women were infected by at least one oncogenic HPV type. Results are consistent with the literature, which indicates that about $50 \%$ of all women are infected by HPV, characterizing a worldwide public health problem [9]. In a previous transverse prevalence study, with a similar population, we found similar results [10].

High-risk oncogenic HPV types has been found to be the main risk factor for cervical neoplasia [9]; it is also a good predictor of subsequent high-grade lesions in young women [11,12]. Corroborating this proposal, HCA II results were strongly associated with the likelihood of SIL, since we observed that $87 \%$ of the patients presenting SIL were also infected by HPV, with a positive trend relating high-risk HPV to cervical transformation $(\mathrm{p}<0.00001)$.

We found that $25 \%$ (1/4) of normal cervical smears hadhigh-risk HPV DNA. This would characterize latent 
Table 1. Age distribution of the human papillomavirus-infected patients

\begin{tabular}{lcccccc}
\hline $\begin{array}{l}\text { Age intervals } \\
\text { (years) }\end{array}$ & $11-20$ & $21-30$ & $31-40$ & $41-50$ & $51-60$ & $61-70$ \\
Number of patients & 149 & 381 & 173 & 59 & 21 & 5 \\
\hline
\end{tabular}

Table 2. Human papillomavirus positivity according to the age of the patients

\begin{tabular}{lcccccc}
\hline $\begin{array}{l}\text { Age intervals } \\
\text { (years) }\end{array}$ & $11-20$ & $21-30$ & $31-40$ & $41-50$ & $51-60$ & $61-70$ \\
Number of patients & 69 & 199 & 87 & 32 & 6 & 2 \\
\hline
\end{tabular}

Table 3. Agreement between the Hybrid Capture Assay and Cytology

\begin{tabular}{lccccc}
\hline \multirow{2}{*}{ Cytology } & \multirow{2}{*}{ N $^{*}$} & \multicolumn{4}{c}{ Hybrid Capture Assay } \\
\cline { 3 - 6 } & & Group A & Group B & Group A/B & Prevalence (\%) \\
\hline Normal & 4 & - & 1 & - & $1(25 \%)$ \\
Inflammatory & 408 & 10 & 49 & 32 & $91(22.3 \%)$ \\
HPV & 284 & 18 & 130 & 75 & $223(78.5 \%)$ \\
$\quad$ LSIL & 88 & 4 & 48 & 24 & $76(86.3 \%)$ \\
$\quad$ HSIL & 4 & - & 4 & - & $4(100 \%)$ \\
Total & 788 & 32 & 232 & 131 & $395(50.1 \%)$ \\
\hline
\end{tabular}

$\mathrm{N}^{*}=$ number of patients.

Table 4. Retrospective analysis of the human papillomavirus patients

\begin{tabular}{lcccc}
\hline \multirow{2}{*}{$\begin{array}{l}\text { Retrospective } \\
\text { analysis of the patients }\end{array}$} & Samples (n) & \multicolumn{3}{c}{ Hybrid Capture Assay } \\
\cline { 3 - 5 } & & GroupA & Group B & Group A/B \\
\hline Regressive lesions & 4 & 1 & 2 & 1 \\
Persistent lesions & 22 & 1 & 18 & 3 \\
Progressive lesions & 2 & - & 1 & 1 \\
Latent infection & 13 & 1 & 10 & 2 \\
Transient infection & 18 & 8 & 7 & 3 \\
Curative treatment & 19 & 15 & 4 & - \\
Total & 78 & 26 & 42 & 10 \\
\hline
\end{tabular}


infections and, as reported by Rosendaal et al., (1996), may mean an increased risk of developing SIL for such infected women [14]. We also found 91 inflammatory smears (with no apparent lesion) that were HPV positive, based on HCA II. These cases could be latent infections or incipient cervical lesions [13], characterizing a low level of HPV gene replication [10]. However, it also might be due to either DNA testing errors or errors in the cytological diagnosis [9,11].

Viral testing could help to focus on a group of patients with a high risk of developing lesions in the near future, especially in Brazil, due to the high frequency of cervical cancer [15]. A positive HCA, even when cytological smears are negative, would determine a closer clinical follow up, with colposcopy being used to confirm the HPV infection and/or the occurrence of SIL, in order to avoid the $10 \%$ to $20 \%$ expected cytological false negatives and false positives [16].

Environmental and host immunological factors may play an important role in the development and outcome of SIL [17]. Several studies have shown that the natural history of a genital HPV infection, including the types and viral load of HPV genomes over time, can influence the risk of SIL, as well as its tendency to regress, persist or progress [18]. Hence, we conducted a retrospective study, analyzing the course of female lesions from 1998 to 2000. We made a retrospective analysis, covering two years, of a limited number of women (78 patients), with repeated HCAII at 12-month intervals (Table IV). Many cases had persistent lesions (28\%), as also found by $\mathrm{Ho}$ et al. [18], who reported nearly $30 \%$ persistence. Persistence of infection with human papillomavirus is thought to have a role in the development of cervical neoplasia [12]. Nevertheless, what determines which infections become persistent is not well known, and it may vary in different populations $[12,20]$. We did not examine HPV viral persistence, which is only achieved by doing a clinical follow-up, including HPV-DNA genotyping. Nevertheless, we were able to evaluate persistent lesions that were left untreated by patients that did not return for clinical follow-up. We also observed that $95.4 \%$ of these patients were positive for high-risk HPV infection, similar to previous studies in which it was suggested that persistent processes caused by oncogenic viruses are required for the development and maintenance of SIL [21,22]. Absence of follow up by the patients, fear of treatment, and misinformation, are problems to be solved by public campaigns that explain the true risks, the probability of dissemination and the importance of treatment. These public measures would not only help control HPV epidemics, but also other sexuallytransmitted diseases. Government health programs for cervical cancer prevention, which have been in course for decades, were intensified from 2002 on; preliminary results indicate a significant decrease in the number of cancer cases, from 50,000 cases to 18,000 cases in 2003 (www.Inca.gov.br). Various epidemiological investigations have already shown that periodic convocation of women is essential to conduct a proper follow up, and this helps explain the program's efficiency [24]. We focused on an intermediate-economic-level female population that had good access to health care; in fact, we did not find cancer cases, corroborating the idea that the victims of cervical cancer are poor women, with a low level of education and limited access to medical care [7].

The natural history of HPV infection is characterized by a very high rate of spontaneous clearance $[18,20]$. We encountered four patients (5.1\%) presenting regressive lesions, 18(23\%) with transient infection and 19(24\%) had successful curative treatment, using either electron cauterization or thrichloroacetic acid (TCA) application.All of the treatments resulted in the elimination of infection, comprising 53\% of the analyzed cases.

High-risk HPV was present in 92\% (12/13) of the latent infections and 56\% (10/18) of the transient processes. Previous studies indicated that $50 \%$ of the HPV transient infections disappear within a few months in most cases, despite the presence of oncogenic virus, probably as a result of a competent host immune response [25,26].

Two patients presented progressive lesions. Highrisk HPV DNA was detected in both cases, reinforcing the idea that high-risk HPV has an etiological role in the development of HSIL. Nevertheless, we observed that there were no cases of cancer in the study population, which leads to the speculation that perhaps 
cancer control is already functioning, at least for our middle class population, which can afford private health care and has good access to information.

Thus, are HCA really necessary to optimize cancer prevention? Due to high costs, HCA is not available in routine clinical practice in poor countries, like ours. We suggest testing only high-risk HPV DNA in cocktail B, which could be sufficient for clinical practice and would reduce the costs of the diagnosis [27]. We also suggest that cervical cancer screening strategies should include HPV testing for persistent lesions or lesions unresponsive to treatment, in order to identify the HPV type and the women with abnormal smears who cannot be properly followed up by cytological methods.

\section{Acknowledgements}

We thankAlexsander Moreira Siqueira for technical assistance. Financial support was provided by a grant from the Conselho Nacional de Pesquisa - CNPq / Brazil.

\section{References}

1. Schiffman M.H. Epidemiology of cervical human papillomavirus infections. Curr Top Microbiol Immunol 1994;186:56-78.

2. Zur Hausen H. Papillomavirus causing cancer: Evasion from host-cell control in early events in carcinogenesis. J Natl Cancer Inst 2000;92(9):690-8.

3. Muñoz N. Human Papillomavirus infection and cancer: the epidemiological evidence. J Clin Virol 2000;19:1-5.

4. Woodworth C.D., Doneger J., Di Paolo J.A. Immortalization of human foreskin keratinocytes by various human papillomavirus DNAs corresponds to their association with cervical carcinoma. J Virol 1989;63:159-64.

5. Schiffman M.H., Brinton L.A. The epidemiology of cervical carcinogenesis. Cancer 1995;76:1888-901.

6. Cox J.T., Lorinczy A.T., Schiffman M.H. Human Papillomavirus testing Hybrid Capture appears to be useful in triaging women with a cytological diagnosis of ASCUS. Am J Obstet Gynecol 1995;172:946-54.

7. Oliveira L.H.S., Rodrigues E.V.M., Lopes A.P.T.A.S.et al. Human papillomavirus 16 detection in cervical lesions, physical state of viral DNA and changes in p53 gene. São Paulo Med J 2003; 121:67-71.
8. Camara G.N.L., Cerqueira D.M., Oliveira A.P.G., et al. Prevalence of Human Papillomavirus types in women with pre-neoplastic and neoplastic cervical lesions in Central Brazil. Mem Inst Oswaldo Cruz 2003;98:122-8.

9. Callaghan J., Karim S., Mortlock S., Winter M., Woodward N. Hybrid Capture as a means of detecting human papillomavirus DNA from liquid-based cytology specimens: a preliminary evaluation. British J of Biom Science 2001;58:184-9.

10. Carvalho M.O.O., Almeida R.W., Leite F.M.S., et al. Detection of Human papillomavirus DNA by the Hybrid Capture assay. B J I D 2003; 7:121-5.

11. Yamazaki H., Sasagawa T., Basha W., et al. Hybrid Capture II and LCR-E7 PCR assays for HPV typing in cervical cytologic samples. Int J Cancer 2001;94:222-7.

12. Kjaer S.K., Van Den BruleA.J.C., Paull G., et al. Type specific persistence of high risk human papillomavirus (HPV) as indicator of high grade cervical squamous intraepithelial lesions in young women: population based prospective follow up study. B M J 2002;325:572-8.

13. Koutsky L.A., Holmes K.K., Critlow C.W.A cohort studyof the risk of cervical neoplasia grade 2 or 3 in relation to papillomavirus infection. $\mathrm{N}$ Engl J Med 1992;327:1272-8.

14. Rozendaal L., Walboomers J.M.M., Van Der Linden J.C., et al. PCR based high-risk HPV in cervical cancer screening gives objective risk assessment of women with cytomorphologically normal cervical smears. Int J Cancer 1996;68:766-9.

15. Kligerman J. Estimativas sobre a incidencia de cancer no Brasil. Rev Bras Canc 2002;48(2):175-9.

16. Clavel C., Masure M., Bory J-P., et al. Hybrid Capture IIbased human papillomavirus detection a sensitive test to detect in routine high grade cervical lesions: a preliminary study on 1518 women. British J Cancer 1999;80(9):1306-11.

17. Moscicki A-B., Palefsky J., Gonzales J., Schoolnick G. K. Human papillomavirus infection in sexually active adolescent female: prevalence and risk factors. Pedatric Res 1990;28;507-13.

18. Ho G.Y.F., Burk R.D., Klein S., et al. Persistent genital human papillomavirus infection as a risk factor for persistent cervical dysplasia. J Nat Cancer Inst 1995;87(18):1365-71.

19. De Brux J., Orth G., Croissant O., et al. Lesions of condylomatuises du col uterin: evolution chez 2466 patients. Bull Cancer 1983;70:410-22.

20. Dillner J. Trends over time in the incidence of cervical neoplasia in comparison to trends over time in human papillomavirus infection. J Clin Virol 2000;19:7-23.

21. Cavalcanti S.M.B, Frugulhetti I.C.P.P, Passos M.R.L, Oliveira L.H.S, Epidemiological aspects of human Papillomavirus infection and cervical cancer in Brazil. J Infection 2000;40:80-7. 
22. Nobbenhuis M.A.E., Walboomers J.M.M., Helmrhorst T.J.M., et al. Relation of human papillomavirus status of cervical lesions and consequences for cervicalcancer screening: a prospective study. The Lancet 1999;354:20-5.

23. Brasil, Instituto Nacional de Câncer - INCa. Estimativas de Patologias tumorais. www.INCa.gov.br - accessed 04/11/2004.

24. Franco e Villa L., Rohan T. Design and methods of the Ludwing-Mc Gill longitudinal study of the natural history of human Papillomavirus infection and cervical neoplasia in Brazil. Pan Am J Public Health 1999;6(4):223-33.

25. Syrjanen K.J., Vayrynen M., Saarikoski S., et al. Natural historyof cervical Human Papillomavirus (HPV) infections based on prospective follow up. Brit J Obstet, Gynecol 1985; 92:1086-92.

26. Langosch K.M., Riethdorf S., Loning T. Association of human papillomavirus infection with carcinoma of the cervix uteri and its precursor lesions: theoretical and pratical implications. Virchows Arch 2000;473:227-33.

27. Syrjänen K.J. Epidemiology of human papillomavirus (HPV) infections and their associations with genital squamous cell cancer. APMIS 1989;97:957-70. 